\title{
The effects of a hospital-based perinatal breastfeeding program on exclusive breastfeeding in Taiwan: a quasi-experimental study
}

\author{
AUTHORS \\ CHING-HSUEH YEH PhD RN ${ }^{1,2}$ \\ YA-PI NG YANG PhD RN ${ }^{3}$ \\ BIH-O LEE PhD RN ${ }^{1}$
}

1 School of Nursing, Kaohsiung Medical University, Kaohsiung, Taiwan

2 Department of Medical Research, Kaohsiung Medical University Hospital, Kaohsiung, Taiwan.

3 Department of Nursing, National Tainan Junior College of Nursing, Tainan, Taiwan

\section{CORRESPONDING AUTHOR}

BIH-O LEE School of Nursing, Kaohsiung Medical University, 100, Shih-Chuan 1st Road, Kaohsiung, 80708, Taiwan. Phone: 886-7-3121101 ext.2625 E-mail: biholee@kmu.edu.tw

\section{ABSTRACT}

Objective: To examine if a perinatal breastfeeding program would improve the exclusive breastfeeding rate at a baby-friendly hospital.

Background: The Ten Steps to Successful Breastfeeding and Baby-Friendly Hospital Initiative have been widely used to improve breastfeeding outcomes worldwide. A hospital-based multi-strategy intervention may provide an opportunity to increase breastfeeding in different countries.

Study design and methods: The study used a quasi-experimental design. Data was collected in a Baby Friendly hospital. A total of 60 mother-infant dyads were included. The experimental group took part in the multi-component perinatal breastfeeding program, while the control group received routine care. The multi-strategy program included prenatal breastfeeding education, birth kangaroo mother care (skin-to-skin contact and non-separation practices) at first breastfeed, continuous 24-hour rooming-in, ongoing kangaroo mother care with breastfeeding on cue, and hospital support visits. The exclusive breastfeeding rate was measured at hospital discharge, and one-month postpartum.
Results: The mothers who participated in the intervention had a greater exclusive breastfeeding rate at hospital discharge and one month postpartum than those in the control group. In the experimental group, $90 \%$ of the infants completed the first feeding within two hours after birth. At discharge, $93.3 \%$ of the mothers in the experimental group and $53.3 \%$ in the control group were exclusively breastfeeding. At one month postpartum, $83.3 \%$ of the mothers in the experimental group and $36.7 \%$ in the control group were still exclusively breastfeeding.

Discussion: The intervention program used in the current study is different to previous studies. The current intervention not only included prenatal education and postpartum support, but also included birth kangaroo mother care at first breastfeed and ongoing kangaroo mother care with breastfeeding on cue. Breastfeeding should be promoted through perinatal comprehensive clinical and social support starting in the prenatal period and continuing through intrapartal, postpartum, and follow-up periods. 
Conclusion: This study was the first study to use a hospital-based multi-strategy intervention including the non-separation of mother-infant dyads and other breastfeeding support for mothers in Taiwan. The program was associated with a significant improvement in the exclusive breastfeeding rate at one month postpartum.

Implication for practice: This study provided initial evidence to support the implementation of mother-infant non-separation practices in improving breastfeeding outcomes. Health professionals can be re-educated regarding birth kangaroo mother care at first breastfeed and ongoing kangaroo mother care with breastfeeding on cue without increasing nursing workloads while infants' fathers and other family members (eg. mothers-in-law/mothers' mothers) can be encouraged to participate in order to achieve family-centred maternity care.

\section{What is already known about the topic?}

- Early and exclusive breast feeding and ongoing breast feeding is the optimal form of nutrition for newborn babies and promotes positive infant and mother outcomes.
- Birth kangaroo mother care plays an important role in providing the optimal atmosphere for a baby's instinctive reflex ability to breastfeed effectively in order to help infant imprinting and suckling.

\section{What this paper adds:}

- The newly developed hospital-based perinatal breastfeeding program has demonstrated effectiveness in promoting exclusive breastfeeding rate at one month postpartum.

- Including birth kangaroo mother care at first breastfeed and ongoing kangaroo mother care with breastfeeding on cue in a perinatal breastfeeding program may lead to more positive breastfeeding experiences for new mothers.

Key Words: birth kangaroo mother care, breastfeeding on cue, exclusive breastfeeding, breastfeeding education.

\section{INTRODUCTION}

In order to be accredited as a baby-friendly hospital, a hospital must demonstrate compliance with the international criteria relating to the Ten Steps for Successful Breastfeeding and the international code of marketing for breast-milk substitutes. ${ }^{1}$ Breastfeeding confers significant health benefits to mother-infant dyads and, consequently, is recommended for all infants worldwide. Exclusive breastfeeding is advocated as the optimal nutrition for infants in the first six months of life. ${ }^{2}$ Breastfeeding is widely known to provide health, social, and economic benefits, and $96.29 \%$ of mother-infant dyads who participated in Taiwan's Baby-Friendly Hospitals Initiative have engaged in breastfeeding; however, only $44.8 \%$ of the infants were still exclusively breastfed at six months in Taiwan-based hospitals. ${ }^{3}$ Overall, the global breastfeeding movement has had a significant impact on increasing initiation of breastfeeding worldwide. However, while breastfeeding initiation rates are high, the duration of exclusive breastfeeding is quite short. In Australia, 93\% of women initiate breastfeeding, but only $66 \%$ still breastfeed at six months, and only $29 \%$ are exclusively breastfeeding at six months. ${ }^{4}$

Globally, the early termination of breastfeeding is commonly due to negative initial breastfeeding experiences and perceived insufficient milk supply, ${ }^{5-7}$ and these factors are primarily related to breastfeeding experiences in the first days after birth. Negative initial breastfeeding experiences might stem in part from the current maternity healthcare practice paradigm of mother-infant separation. Two cross-sectional surveys in Poland illustrated the effects of separation versus non-separation of mother-infant dyads immediately after birth on breastfeeding outcomes. ${ }^{8,9}$ Mazur, Mikiel-Kostyra, and their associates have identified various factors in maternity hospital care affecting exclusive breastfeeding. ${ }^{8}$ The most significant factors in hospital care relating to decreased initiation, continuation, and exclusivity were breastfeeding initiation more than two hours postbirth, a lack of skin-to-skin contact, and mother-infant separation lasting longer than 24 hours.

Previous research has suggested the use of the following five interventions to facilitate the continuation of exclusive breastfeeding: prenatal breastfeeding education (PBE) for parents, ${ }^{10-12}$ birth kangaroo mother care (BKMC) at first breastfeed, ${ }^{1}$ continuous 24 -hour rooming-in, ${ }^{13-15}$ ongoing kangaroo mother care (KMC) with breastfeeding on cue, ${ }^{16}$ and support from health professionals. ${ }^{17}$ Therefore, it is important that non-separation practice (that is, skin-toskin contact) is applied from birth to discharge to promote breastfeeding outcomes. It is also important for multistrategy interventions to encourage parents to maintain exclusive breastfeeding for an extended duration. That said, as we found no previous studies utilising the intervention 
we used in the Taiwanese study, we consider this to be the first study in Taiwan to use an intervention combining "BKMC until the first breastfeed is completed" with other key strategies to promote an extended duration of exclusive breastfeeding.

\section{BACKGROUND}

Prenatal breastfeeding education (PBE) for parents is important as an effective single intervention for the promotion of breastfeeding. One of the psychosocial factors commonly affecting the early termination of breastfeeding is the lack of support from the father of the infant. ${ }^{18}$ Consequently, infants' fathers need to participate in PBE in order to better support breastfeeding. A systematic review and meta-analysis provided evidence that PBE increases the initiation of breastfeeding and the short-term duration of breastfeeding, although it did not have a significant impact on the long-term duration of exclusive breastfeeding. ${ }^{10,11,19}$ One early breastfeeding program reviewed in a previous study focused on the benefits of breastfeeding, traditional hands-on breastfeeding skills, and getting started. ${ }^{20}$ However, the program did not provide any new information on the importance of the concept of mother-infant dyad nonseparation or infant-led breastfeeding skills. ${ }^{21}$ Rather, the program was designed to provide more general PBE and psychological preparation for parents.

The classifications of the stages of kangaroo mother care (KMC) are as follows: birth, very early, early, intermediate, and late stage. ${ }^{22}$ Birth kangaroo mother care (BKMC) and very early kangaroo mother care (VEKMC) initially occur in the delivery room. BKMC begins immediately (within one minute) post-birth and before cutting of the umbilical cord. VEKMC begins after finishing initial infant care such as pharyngeal suction, Apgar score, drying of head and skin, and cord care - usually between 20 and 30 minutes post-birth. Early KMC occurs from the first hour to the first week postbirth and is done with infants who are easily stabilised and begins as soon as they are stable post-birth. Intermediate KMC usually begins after seven days post-birth when the infants have completed early intensive care, still require oxygen and may have apnea and bradycardia. Late KMC usually occurs many weeks post-birth when the infants have completed the intensive care phase, have stable respiration, and are breathing room air.22

The non-separation paradigm is defined as mother-infant dyads remaining together from the first minute after birth (called birth kangaroo mother care, or BKMC) until hospital discharge (called ongoing kangaroo mother care, or KMC). BKMC is defined as mothers holding their naked and fullterm infants upright and prone, with skin-to-skin contact, on the mothers' abdomen or chest during the first minute after birth. ${ }^{23}$ As a part of BKMC, infants are allowed to breast crawl $^{24-26}$ and have self-regulatory ${ }^{27}$ access to breastfeeding.
This is called "BKMC at first breastfeed." BKMC is the best practice for providing opportunities for the first feeding, ${ }^{28}$ facilitating mother-infant bonding, facilitating mutual physiological adaptation, and encouraging longer durations of exclusive breastfeeding. ${ }^{29-31}$ BKMC plays an important role in providing the optimal atmosphere for the instinctive reflex ability to breastfeed effectively in order to improve breastfeeding outcomes.

The definition of ongoing KMC is that full-term infants have skin-to-skin contact (SSC) with their mothers beginning within the first minute after birth and continuing as much as possible throughout the postpartum period. SSC involves the infant, wearing only a diaper, being placed between the mother's breasts and then covered by a blanket. Ongoing KMC is initiated in a hospital and can be continued at home. Evidence for ongoing KMC includes its effect on three major aspects of mother-infant dyads: (a) bonding; ;2,33 (b) physiology, such as thermoregulation ${ }^{34}$ and analgesia; 35 (c) increasing parental sensitivity to infant cues; 33 and (d) breastfeeding initiation, duration, and exclusivity. ${ }^{31}$ Ongoing KMC is the best practice for providing an opportunity for mothers to learn pre-feeding behaviour. BKMC at first breastfeed and ongoing KMC with breastfeeding on cue are critical for effective latch-on and suckling. ${ }^{28}$ An ideal time for first latch-on occurs during BKMC, when infants use their sense of smell to locate the breast and nipple. Mothers learn the signs of prefeeding behaviour from engaging in KMC. Ongoing KMC helps infants to stay calm and quiet and facilitates the initiation, continuation, and exclusivity of breastfeeding. When mothers are encouraged to breastfeed on cue, they need support from health professionals and family members.

Health professional support ${ }^{36}$ and support from the infant's father ${ }^{37}$ are beneficial and valuable to the initiation and continuation of breastfeeding. Mother-infant dyads need breastfeeding support from the infants' fathers and health professionals prior to hospital discharge so that the mothers can go home confident of adequately nourishing their infants, establish and maintain a rich milk supply, and avoid psychophysiological breastfeeding problems (such as the delayed onset of lactation and perceived insufficient milk supply). Interventions combining PBE with health professional support were the most common interventions for improving breastfeeding intentions and outcomes. ${ }^{38,39}$ However, no combined interventions were found in the previous studies conducted in Taiwan. Our study thus incorporated the various international recommendations on PBE for parents, BKMC at first breastfeed, continuous 24-hour rooming-in, ongoing KMC with breastfeeding on cue, and health professionals' support into a single program, which we called a perinatal breastfeeding program (PBP), for healthy primiparous mothers who gave birth vaginally to healthy full-term infants at a baby-friendly hospital in southern Taiwan. The purpose of the study was to examine the 
effectiveness of this PBP in terms of improving exclusive breastfeeding rates at hospital discharge, and one-month postpartum.

\section{METHODS}

The study used a quasi-experimental design. In order to minimise contamination across groups, the study first investigated a control group that received routine care before then investigating the experimental group that took part in the PBP. The study included healthy and pregnant women in the third trimester of their first pregnancy who were receiving regular prenatal care. The participants were recruited before giving birth using several criteria:

(a) primigravida status, (b) vaginal delivery, and

(c) willingness to breastfeed. However, they were excluded if they had (a) pre-existing medical problems or

(b) complications with their pregnancy such as preeclampsia or eclampsia. After birth, a second check of eligibility for the study was conducted in the delivery room. The mothers and infants were included in this check. The infant inclusion criteria were: (a) singleton status, (b) a 1-min Apgar score of 7 or greater, and (c) 37-42 weeks of gestation at birth. The criteria of (b) and (c) define a healthy full-term infant, which was a prerequisite for doing BKMC. Potential participants were excluded based on the conditions of separation of infant-mother dyads that interfered with the initiation of breastfeeding such as: (a) mother had postpartum complications or (b) infant had a congenital disease or an acute illness.

This study was conducted at a baby-friendly medical centre in southern Taiwan. Primigravida women who met the inclusion criteria were contacted by the researcher at 34-37 weeks' gestation for a face-to-face interview at a regularly scheduled prenatal clinic appointment. The study used a nonprobability, convenience sample of third trimester primigravida women. For sample size calculation, a medium effect size was set at 0.3. The alpha was set at 0.05 , power at $\mathrm{o} .8 \mathrm{o}$, and number of repetition at 2. We used G-Power version 3.1, applying the F-test approach with a $20 \%$ drop-out rate..$^{40}$ At least 56 mother-infant dyads were needed.

The maternal prenatal demographic questionnaire (MPDQ) included the mother's age, education level, occupation, parenting leave, whether the mother attended group breastfeeding classes, and the father's support for breastfeeding. The BKMC log included the timing of SSC, duration of KMC, timing of first feeding, and duration of the first breastfeeding session. A KMC log was provided to the mothers to record the frequency and duration of KMC. The researcher obtained the total hours of rooming-in from nursing records. The Index of Breastfeeding Status (IBS) was used to measure the exclusive breastfeeding rate. The IBS is a self-report instrument used to measure the pattern and incidence of breastfeeding status. The IBS has the following categories: exclusive breastfeeding, almost exclusive breastfeeding, high breastfeeding, partial breastfeeding, token breastfeeding, and formula feeding. ${ }^{41}$ Exclusive breastfeeding is defined as 100\% human milk and no other liquid or solids given to the infant. Almost exclusive breastfeeding is defined as human milk plus vitamins, minerals, water, or juice given infrequently. High breastfeeding is defined as above $80 \%$ human milk with one or less than one formula feeding per day. Partial breastfeeding is defined as more than one formula feeding per day. Token breastfeeding refers to breastfeeding for comforting the infant and not for nutritional reasons. Formula feeding refers to no breastfeeding. If the mothers chose to mix feeding or stop breastfeeding their infants, they were asked when they started mixing or stopped breastfeeding, and what the reasons for the decision were. ${ }^{41}$

The BKMC log and KMC log recorded the implementation of the intervention. The IBS recorded the classification of the infant feeding and collected the qualitative data, such as a reason for formula feeding. Therefore, the reliability of the instruments for variables are not applicable. The MPDQ was completed during the aforementioned prenatal visit. MPDQ (prenatal clinics), BKMC log (after birth immediately), and IBS (before hospital discharge and one month postpartum) data were collected from both groups, while KMC log data were only collected from the postpartum experimental group.

\section{THE PBP AS INTERVENTION}

The PBP is a theoretically based intervention used to provide $\mathrm{PBE}$ and postpartum breastfeeding support for the parents. The control group was studied first and received routine care. When data collection for the control group was completed, 26 nursing and medical staff were instructed about the intervention in a one hour session. The training course included information on the PBP, particularly focused on BKMC at first breastfeed and ongoing KMC with breastfeeding on cue. Appendix A (see Supplementary Data File) details how the PBP used the Prenatal-IntrapartumPostpartum-Follow-up model to guide the study. Appendix B (see Supplementary Data File) shows the differences between routine care (control group) and the PBP (experimental group).

Each of the couples in the experimental group participated in a 9o-min one-on-one PBE session before birth. They also received a booklet with an audio-video CD-ROM titled "New Idea of Successful Breastfeeding” (Image 1, Supplementary Data File) that was developed by the researcher. The contents of the booklet and the supporting audio-video CD-ROM were validated by three health professionals and three mothers who had breastfeeding experience. All of the couples were asked to read the booklet and watch the video before the PBE class. The PBE was implemented during the third trimester of pregnancy. 
The researchers provided a kangaroo sling to each of the experimental mother-infant dyads for safety during ongoing KMC, as well as a booklet to guide the mother on how to engage in ongoing KMC and how to monitor the safety of the ongoing KMC (Image 2, Supplementary Data File).

\section{DATA ANALYSIS}

The research hypothesis was that mothers who participate in the PBP will have a greater incidence of exclusive breastfeeding when compared to those in the control group at the time of hospital discharge and one month postpartum. Data were coded and analysed by using SPSS version 20.0 for Windows. The MPDQ, BKMC log and KMC log data were analysed using the independent $t$ test, Chi-square test and
Fisher's exact test. The IBS data were analysed using the Chi-square test and Fisher's exact test. Descriptive statistics, including frequencies, means, and standard deviations, were used to examine the demographic characteristics. The independent t test, Chi-square test, and Fisher's exact test were used to analyse differences between the two groups.

\section{ETHICAL CONSIDERATIONS}

With respect to ethical considerations, the study was approved by the Institutional Review Board in the study hospital (IRB number: KMUH-IRB-980o62). All of the participants agreed to participate and signed an informed consent form.

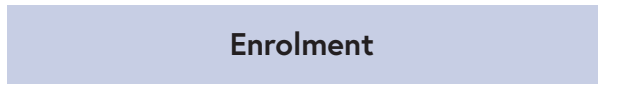

1st Assessed for eligibility $(n=103)$

Excluded $(n=13)$

- Not meeting inclusion criteria $(n=0)$

- Refused to participate $(n=13)$

- Other reasons $(n=0)$

Non-Randomised $(n=90)$

The control group was studied first, followed by the experimental group

Allocated to control group $(n=46)$

- Received usual care $(n=46)$
Allocated to experimental group $(n=44)$

- Received PBP program $(n=44)$

\section{2nd Assess for Eligibility after Birth}

Discontinued intervention $(n=16)$

- Caesarean birth $(n=11)$

- Mother-infant separation $(n=4)$

- Infant death $(n=1)$
Discontinued intervention $(n=14)$

- Caesarean birth $(n=9)$

- Mother-infant separation ( $n=5)$

- Infant death $(n=0)$

\section{Follow-Up 1 month}

Lost to follow-up 1 month postpartum $(n=0)$
Lost to follow-up 1 month postpartum $(n=0)$

\section{to follow-up 1 month postpartum $(n=0)$}

\section{Analysis}

Analysed $(n=30)$
Analysed $(n=30)$ 


\section{RESULTS}

A total sample of 103 pregnant mothers was recruited. Thirteen of these potential participants declined participation and the remaining potential participants were assigned into the control group (CG, $n=46$ ) or the experimental group (EG, $n=44$ ). Then, at the second assessment of eligibility after birth, 30 potential participants were excluded due to having cesarean birth $(n=20)$, infant death $(n=1)$ or mother-infant separation $(n=9)$, leaving a final total of 60 participants (CG, $n=30$; EG, $n=30$ ) who were included in the study (Figure 1).
Ninety percent of the infants' fathers were very supportive or supportive of breastfeeding in the MPDQ (Table 1). With the exception of taking prenatal group breastfeeding classes ( $p=.029)$, there were no significant differences between the experimental group and the control group with regard to the demographic variables. This difference reflected the fact that a greater number of mothers in the control group (43.3\% vs. 16.7\%) had taken a prenatal group breastfeeding class (Table 1).

\section{TABLE 1: PRENATAL DEMOGRAPHIC CHARACTERISTICS}

\begin{tabular}{|c|c|c|c|c|c|c|}
\hline \multirow[t]{2}{*}{ Measure } & \multirow{2}{*}{$\begin{array}{c}\text { Total Group } \\
N=60 ; M(S D)\end{array}$} & \multirow{2}{*}{$\begin{array}{c}\text { PBP Group } \\
n=30 ; M(S D)\end{array}$} & \multirow{2}{*}{$\begin{array}{l}\text { Control Group } \\
n=30 ; M(S D)\end{array}$} & \multicolumn{3}{|c|}{ Statistics } \\
\hline & & & & $x^{2}$ & $t$ & $p$ \\
\hline \multirow[t]{2}{*}{ Maternal age (years) } & $31.25(3.28)$ & $31.33(3.68)$ & 31.17 (2.89) & & -.20 & .85 \\
\hline & $n(\%)$ & $n(\%)$ & $n(\%)$ & $x^{2}$ & $t$ & $p$ \\
\hline \multicolumn{7}{|c|}{ Maternal education level } \\
\hline$\leq 12$ years & $10(16.7)$ & $03(10.0)$ & $07(23.3)$ & $1.97^{a}$ & & .30 \\
\hline$>12$ years & $50(83.3)$ & $27(90.0)$ & $23(76.7)$ & & & \\
\hline \multicolumn{7}{|l|}{ Maternal occupation } \\
\hline Non-medical & $32(53.3)$ & $14(46.7)$ & $18(60.0)$ & 1.07 & & .30 \\
\hline Medical & $28(46.7)$ & $16(53.3)$ & $12(40.0)$ & & & \\
\hline \multicolumn{7}{|l|}{ Parenting leave } \\
\hline \multicolumn{7}{|c|}{ Group breastfeeding classes } \\
\hline Yes & $18(30.0)$ & $05(16.7)$ & $13(43.3)$ & 5.08 & & $.029 *$ \\
\hline \multicolumn{7}{|c|}{ Paternal breastfeeding support } \\
\hline Neutral & $06(10.0)$ & $03(10.0)$ & $03(10.0)$ & $.44^{a}$ & & .93 \\
\hline Supportive & $18(30.0)$ & $10(33.3)$ & $08(26.7)$ & & & \\
\hline Very supportive & $36(60.0)$ & $17(56.7)$ & $19(63.3)$ & & & \\
\hline
\end{tabular}

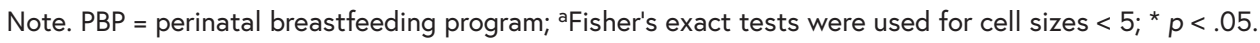

\section{TABLE 2: OUTCOMES OF INTRAPARTUM-POSTPARTUM INTERVENTION}

\begin{tabular}{|c|c|c|c|c|c|}
\hline \multirow[t]{2}{*}{ Measure } & \multirow{2}{*}{$\begin{array}{c}\text { PBP Group } \\
n=30 ; M(S D)\end{array}$} & \multirow{2}{*}{$\begin{array}{l}\text { Control Group } \\
n=30 ; M(S D)\end{array}$} & \multicolumn{3}{|c|}{ Statistics } \\
\hline & & & $x^{2}$ & $t$ & $p$ \\
\hline Timing of SSC (hr:min) at birth & $00: 02(0: 03)$ & $00: 14(0: 04)$ & & 13.09 & $.00 * \star *$ \\
\hline Duration of SSC (hr:min) ${ }^{b}$ at birth & $01: 19(0: 21)$ & 01:06 (0:25) & & -2.23 & $.02^{\star}$ \\
\hline Timing of first feeding (hr:min) ${ }^{b}$ & $01: 33(1: 40)$ & 07:09 (8:43) & & 3.34 & $.00^{\star \star}$ \\
\hline Rooming-in hours (hr:min) & $19: 55(7: 19)$ & $20: 10(5: 45)$ & & .15 & .88 \\
\hline \multirow[t]{2}{*}{ Duration of KMC (hr:min) } & $06: 52(4: 26)$ & $00: 00(0: 00)$ & & -8.45 & $.00^{\star \star \star}$ \\
\hline & $n(\%)$ & $n(\%)$ & $x^{2}$ & $t$ & $p$ \\
\hline \multicolumn{6}{|l|}{ First feeding $\leq 2$ hrs. after birth } \\
\hline Yes & $27(90.0)$ & $04(13.3)$ & $40.05^{a}$ & & $.00^{\star \star \star}$ \\
\hline \multicolumn{6}{|l|}{ KMC (Day 2) } \\
\hline
\end{tabular}

Note. PBP = perinatal breastfeeding program; SSC = skin-to-skin contact; KMC = kangaroo mother care.

${ }^{a}$ Fisher's exact tests were used for cell sizes $<5$. bMissing data; ${ }^{\star} p<.05 .{ }^{\star \star} p<.01 .{ }^{\star \star \star} p<.001$. 
With the exception of the duration of rooming-in hours, there were statistically significant differences in the implementation of the interventions included in the PBP, reflecting the fact that the control group did not receive the PBP intervention (Table 2).

In the experimental group, 90\% of the infants completed the first feeding within two hours after birth. There was a statistically significant difference between the two groups in the timing of the first breastfeeding $\left(t=3.34, p<.01 ; x^{2}=40.05\right.$, $p<.001)$.

The numbers of mothers continuing to breastfeed in the total study cohort, the control group, and the experimental group are presented in Table 3.

TABLE 3: INDEX OF BREASTFEEDING STATUS (IBS)

\begin{tabular}{|c|c|c|c|c|}
\hline \multirow[t]{2}{*}{ Measure } & \multirow{2}{*}{$\begin{array}{l}\text { PBP Group } \\
n=30 ; n(\%)\end{array}$} & \multirow{2}{*}{$\begin{array}{l}\text { Control Group } \\
n n=30 ; n(\%)\end{array}$} & \multicolumn{2}{|c|}{ Statistics } \\
\hline & & & $x^{2}$ & $P$ \\
\hline \multicolumn{5}{|l|}{ IBS $\left(T_{1}\right)$} \\
\hline Exclusive & $28(93.3)$ & $16(53.3)$ & $13.32^{\mathrm{a}}$ & $.00^{\star \star}$ \\
\hline High & $01(3.3)$ & $02(6.7)$ & & \\
\hline Partial & 01 (3.3) & $11(36.7)$ & & \\
\hline Token & $00(.0)$ & 01 (3.3) & & \\
\hline \multicolumn{5}{|l|}{ IBS $\left(T_{2}\right)$} \\
\hline Exclusive & $25(83.3)$ & $11(36.7)$ & $15.95^{a}$ & $.00^{\star \star \star}$ \\
\hline High & 03 (10.0) & 04 (13.3) & & \\
\hline Partial & 02 (6.7) & $14(46.7)$ & & \\
\hline Formula & $00(.0)$ & $01(3.3)$ & & \\
\hline
\end{tabular}

Note. $\mathrm{PBP}=$ perinatal breastfeeding program; $\mathrm{T}_{1}=$ discharge data collection; ${ }^{* \star} p<.01 .{ }^{* * *} p<.001$.

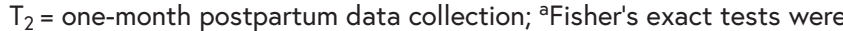
used for cell sizes $<5$.

At discharge, 93.3\% of the mothers in the experimental group and $53.3 \%$ in the control group were exclusively breastfeeding. At one month postpartum, $83.3 \%$ of the mothers in the experimental group and $36.7 \%$ in the control group were still exclusively breastfeeding. These differences were statistically significant $\left(x^{2}=13.32, p<.01\right.$ at $\mathrm{T}_{1} ; x^{2}=15.95, p<.001$ at $\left.\mathrm{T}_{2}\right)$.

\section{DISCUSSION}

This study provides initial evidence to support the implementation of mother-infant non-separation practices (that is, SSC) in improving breastfeeding outcomes in Taiwan. In Taiwan, all baby-friendly hospitals provide very early KMC, with the first mother-infant dyad contact following the completion of initial infant care, and most infants have their first breastfeeding within four hours of birth. Early maternal-newborn contact not only improves the birth experience ${ }^{42}$ but also enhances breastfeeding outcomes. ${ }^{13}$ The approach used in this study was different from the routine care, in that in BKMC, the mother-infant SSC began in the first minutes after birth and lasted until the first breastfeed. It was also different from the approaches used in other studies as it emphasised innate mutual self-regulation between the mother and infant via non-separation while the dyad remained hospitalised. This st udy encouraged the mothers to practice not only continuous 24-hour roomingin, but also ongoing KMC with breastfeeding on cue. While as a single intervention prenatal breastfeeding education may be an effective approach, 43 the PBP is a hospitalbased multi-strategy intervention to promote ongoing breastfeeding outcomes post-partum. The experimental group of mothers in this study had a greater incidence of exclusive breastfeeding compared to the control group, which is consistent with the prior research. ${ }^{28,39}$ In this study, $83.3 \%$ of the experimental group and $36.7 \%$ of the control group mothers were exclusively breastfeeding at one month post-birth. In comparison, in Kupratakul et al.'s RCT study, $77.5 \%$ of the experimental group of mothers $(n=40)$ and $52.6 \%$ of control group of mothers $(n=40)$ were exclusively breastfeeding at one month post-birth. ${ }^{39}$ Kupratakul's study was conducted in Bangkok, Thailand, using the knowledgesharing practices with empowerment strategies (KSPES) program for prenatal education and postpartum support strategies to improve the rates of exclusive breastfeeding. The PBP used in the current study is different from the KSPES program, as the PBP not only included prenatal education and postpartum support, but also BKMC at first breastfeed and ongoing KMC with breastfeeding on cue.

These findings suggest that the additional prenatal education and postpartum support strategies used in the present study may help improve the rates of exclusive breastfeeding at one month postpartum. Most of the infants in this study were assisted in latching on to the breast correctly by the researcher. Only two of the infants were able to crawl up to the breast, self-latch, and suckle. As such, BKMC until the first breastfeeding was completed had a significant impact on the breastfeeding outcomes. This study on BKMC with first feeding was similar to that of Widstrom's with regard to early optimal self-regulation, and this phenomenon impacts the exclusive breastfeeding rate. ${ }^{28}$

The most influential factors are maternal breastfeeding intention and knowledge, as well as social support from family members or health professionals. ${ }^{19}$ Breastfeeding should be promoted through perinatal comprehensive clinical and social support starting in the prenatal period and continuing through the intrapartum, postpartum, and follow-up periods. This suggests that efforts should be made in PBPs to help women obtain positive initial breastfeeding experiences to increase the rate of exclusive breastfeeding. 


\section{LIMITATIONS}

This study had some limitations. The telephone follow-ups were similar between the two groups because the researcher found it difficult to refuse to answer breastfeeding questions from those in the control group. Therefore, the control group were, at least partially, supported for breastfeeding after going home. This could have affected research outcomes. Many participants were not familiar with KMC and so refused to participate. As such, generalisation of the results of this study to the general population should be done with caution, as KMC may have varying acceptability among different populations. Ongoing KMC was hindered by the family members, especially mothers-in-law. The mothers-inlaw were worried that the mother or infant would catch a cold while doing KMC. Traditional Taiwanese practices after birth (for the first month) include the mother staying at home, being taken care of by others, eating a special diet, and obeying traditional rituals. The introduction of KMC, with which mothers-in-law are generally not familiar, could have been facilitated in the study by including them in prenatal education, as will be done in future studies. Multicentre studies should be performed to ensure increased sample sizes and, potentially, more representative samples of the target population, as this could increase the generalisability of future studies. Further research is needed to measure duration of exclusive breastfeeding up to six months postbirth among different populations, to determine if duration is consistent with WHO recommendations. Moreover, other outcomes such as the mother's satisfaction with the experience could be measured in future studies.

\section{CONCLUSION}

The findings provide early evidence to support the implementation of a PBP in improving the exclusive breastfeeding rate. The results of this study supported the BFHI launched by the WHO and UNICEF to Step 4: "Place babies in skin-to-skin contact with their mothers immediately following birth for at least an hour. Encourage mothers to recognise when their babies are ready to breastfeed and offer help if needed"1(p34) The results also supported the Academy of Breastfeeding Medicine Committee guidelines that "the healthy infant can be given directly to the mother for skin-to-skin contact until the first feeding is accomplished," and that "the initial physical assessment [should be] performed as the infant is placed with the mother". Future studies may conduct group PBE classes, which are more feasible and would decrease the nursing workload. In summary, the promotion of successful exclusive breastfeeding requires multi-strategy nursing interventions to overcome individual differences. The findings from this study provide preliminary evidence to support the implementation of mother-infant non-separation practices in improving breastfeeding outcomes.
Acknowledgements: Thanks are due to the dyads and infants' fathers who participated in the study, and the obstetrics medical and nursing staff of Kaohsiung Medical University Hospital for help in the successful completion of the study.

Conflict of interest: There is no conflict of interest regarding the publication of this article.

Use of images: The author maintains express permission to publish the images presented in this research paper.

Funding sources: The research was not funded.

\section{REFERENCES}

1. United Nations Children's Fund \& World Health Organization. Baby-friendly hospital initiative: Revised, updated and expanded for integrated care, section 1, background and implementation: original BFHI guidelines developed 1992. 2009. http://www.who.int/nutrition/topics/BFHI Revised Section1. pdf (accessed 30 Mar 2020).

2. Ho C. Optimal duration of exclusive breastfeeding. International Journal of Evidence-Based Healthcare. 2013; 11(2): 140-1.

3. Health Promotion Administration. Breastfeeding current status in Taiwan (Traditional Chinese). 2017. https://www.hpa.gov. tw/Pages/Detail.aspx?nodeid $=506 \&$ pid=463 (accessed 4 Feb 2019).

4. Australian Bureau of Statistics. National Health Survey: first results 2017-18. 2019. https://www.abs.gov.au/ausstats/abs@. nsf/Lookup/by\%20Subject/4364.0.55.001 2017-18 Main\%20 Features Breastfeeding 10019 (accessed 30 Mar 2020).

5. DiGirolamo AM, Thompson N, Martorell R, Fein S, GrummerStrawn L. Intention or experience? predictors of continued breastfeeding. Health Educ Behav. 2005; 32(2): 208-26.

6. Lou Z, Zeng G, Huang L, Wang Y, Zhou L, Kavanagh KF. Maternal reported indicators and causes of insufficient milk supply. J Hum Lact. 2014; 30(4): 466-73.

7. Dietrich Leurer M, Misskey E. "Be positive as well as realistic": a qualitative description analysis of information gaps experienced by breastfeeding mother. Int Breastfeed J. 2015; 10(1): 10

8. Mazur J, Mikiel-Kostyra K. Determinants of newborn feeding in maternity hospital care. Part II: factors associated with exclusive breastfeeding (Polish). Ginekol Pol. 2000; 71(7): 604-10.

9. Mikiel-Kostyra K, Mazur J, Wojdan-Godek E. Factors affecting exclusive breastfeeding in Poland: cross-sectional survey of population-based sample. Soz Praventivmed. 2005; 50(1): 52-9.

10. Brown A, Davies R. Fathers' experiences of supporting breastfeeding: challenges for breastfeeding promotion and education. Matern Child Nutr. 2014; 10(4): 510-26.

11. Wong KL, Tarrant M, Lok KYW. Group versus individual professional antenatal breastfeeding education for extending breastfeeding duration and exclusivity. J Hum Lac. 2015; 31(3): 354-66.

12. Yeh $\mathrm{CH}$. Health Education for Successful Breastfeeding Formosan J Med. 2015; 19(6): 643-9. 
13. Chiou ST, Chen LC, Yeh H, Wu SR, Chien LY. Early skin-to-skin contact, rooming- in, and breastfeeding: a comparison of the 2004 and 2011 national surveys in Taiwan. Birth. 2014; 41(1): 338.

14. Dumas L, Lepage M, Bystrova K, Matthiesen A-S, WellesNyström B, Widström A-M. Influence of skin-to-skin contact and rooming-in on early mother-infant interaction: a randomized controlled trial. Clin Nurs Res. 2013; 22(3): 310-36.

15. McCoy RC, Chantry CJ, Howard CR, Lawrence RA, Marinelli KA, Powers NG. ABM clinical protocol \#5: peripartum breastfeeding management for the healthy mother and infant at term revision. Breastfeed Med. 2008; 3(2): 129-32.

16. World Health Organization. Kangaroo mother care: a practical guide. 2003. http://www.who.int/making pregnancy safer/ documents/9241590351/en/ (accessed 15 Oct 2014).

17. Rosin SI, Zakarija-Grković I. Towards integrated care in breastfeeding support: a cross-sectional survey of practitioners' perspectives. Int Breastfeed J. 2016; 11(1): 1-17.

18. Wallenborn JT, Masho SW, Ratliff S. Paternal pregnancy intention and breastfeeding duration: findings from the national survey of family growth. Matern Child Health J. 2017; 21(3): 554-61.

19. Wouk K, Tully KP, Labbok MH. Systematic review of evidence for baby-friendly hospital initiative step 3. J Hum Lact. 2017; 33(1): 50-82.

20. Huang MZ, Kuo SC, Avery MD, Chen W, Lin KC, Gau ML. Evaluating effects of a prenatal web-based breastfeeding education programme in Taiwan. J Clin Nurs. 2007; 16(8): 15719.

21. Brown A, Arnott B. Breastfeeding duration and early parenting behaviour: the importance of an infant-led, responsive style. PLoS One. 2014; 9(2): e83893.

22. Anderson GC. Touch and the kangaroo care method. In: Field TM, ed. Touch in early development. Mahwah, NJ: Lawrence Erlbaum Associates, 1995; 35-51.

23. Moore ER, Anderson GC. Randomized controlled trial of very early mother-infant skin-to-skin contact and breastfeeding status. J Midwifery Womens Health. 2007; 52(2): 116-25.

24. Gangal P, Bhagat K, Prabhu S, Nair R. Breast crawl: initiation of breastfeeding by breast crawl. 2007. http://breastcrawl.org/ pdf/breastcrawl.pdf (accessed 12 Mar 2018).

25. Righard L, Alade MO. Effect of delivery room routines on success of first breast-feed. Lancet. 1990; 336(8723): 1105-7.

26. Widstrom AM, Ransjo-Arvidson AB, Christensson K, Matthiesen AS, Winberg J, Uvnas-Moberg K. Gastric suction in healthy newborn infants: effects on circulation and developing feeding behaviour. Acta Paediatr Scand. 1987; 76(4): 566-72.

27. Anderson GC. Risk in mother-infant separation postbirth. Image J Nurs Sch. 1989; 21(4): 196-9.

28. Widstrom AM, Lilja G, Aaltomaa-Michalias P, Dahllof A, Lintula M, Nissen E. Newborn behaviour to locate the breast when skin-to-skin: a possible method for enabling early selfregulation. Acta Paediatr. 2011; 100(1): 79-85.

29. Lowson K, Offer C, Watson J, McGuire B, Renfrew MJ. The economic benefits of increasing kangaroo skin-to-skin care and breastfeeding in neonatal units: analysis of a pragmatic intervention in clinical practice. Int Breastfeed J. 2015; 10: 11.

30. Moore ER. Early skin-to-skin contact for mothers and their healthy newborn. J Obstet Gynecol Neonatal Nur. 2013; 42(s1): s86.
31. Moore ER, Anderson GC, Bergman N. Early skin-to-skin contact for mothers and their healthy newborn infants. Cochrane Database Syst Rev. 2007; (3): Cd003519.

32. Klaus MH. Mother and infant: early emotional ties. Pediatrics. 1998; 102(5 Suppl E): 1244-6.

33. Nyqvist KH, Anderson GC, Bergman N, Cattaneo A, Charpak $N$, Davanzo R, et al. Towards universal kangaroo mother care: recommendations and report from the first European conference and seventh international workshop on kangaroo mother care. Acta Pædiatr. 2010; 99(6): 820-6.

34. Bystrova K, Widstrom AM, Matthiesen AS, Ransjo-Arvidson AB, Welles-Nystrom B, Wassberg C, et al. Skin-to-skin contact may reduce negative consequences of "the stress of being born": a study on temperature in newborn infants, subjected to different ward routines in St. Petersburg. Acta Paediatr. 2003; 92(3): 320-6.

35. Chermont AG, Falcao LF, de Souza Silva EH, de Cassia Xavier Balda R, Guinsburg R. Skin-to-skin contact and/or oral 25\% dextrose for procedural pain relief for term newborn infants. Pediatrics. 2009; 124(6): e1101-7.

36. Hannula L, Kaunonen M, Tarkka MT. A systematic review of professional support interventions for breastfeeding. J Clin Nurs. 2008; 17(9): 1132-43.

37. Maycock B, Binns CW, Dhaliwal S, Tohotoa J, Hauck Y, Burns S, et al. Education and support for fathers improves breastfeeding rates: a randomized controlled trial. J Hum Lact. 2013; 29(4): 484-90.

38. Parry KC, Tully KP, Hopper LN, Schildkamp PE, Labbok MH. Evaluation of Ready, Set, BABY: a prenatal breastfeeding education and counseling approach. Birth. 2019; 46(1): 113-20.

39. Kupratakul J, Taneepanichskul S, Voramongkol N, Phupong V. A randomized controlled trial of knowledge sharing practice with empowerment strategies in pregnant women to improve exclusive breastfeeding during the first six months postpartum. J Med Assoc Thai. 2010; 93(9): 1009-18.

40. Heinrich-Heine-University Düsseldorf. G*Power: statistical power analyses for Windows and Mac. 2017. http://www.psychologie.hhu.de/arbeitsgruppen/allgemeinepsychologie-und-arbeitspsychologie/gpower.html (accessed 10 Jan 2019).

41. Labbok MH, Krasovec K. Toward consistency in breastfeeding definitions. Stud Fam Plann. 1990; 21(4): 226-30.

42. Brubaker LH, Paul IM, Repke JT, Kjerulff KH. Early maternalnewborn contact and positive birth experience. Birth. 2019; 46(1): 42-50.

43. Guise JM, Palda V, Westhoff C, Chan BK, Helfand M, Lieu TA. The effectiveness of primary care-based interventions to promote breastfeeding: systematic evidence review and metaanalysis for the US preventive services task force. Ann Fam Med. 2003; 1(2): 70-8. 